\title{
Abstract: Reduktion der Kalibrierungszeit für die Magnetpartikelbildgebung mittels Deep Learning
}

\author{
Ivo M. Baltruschat ${ }^{1,2}$, Patryk Szwargulski ${ }^{1,2}$, Florian Griese ${ }^{1,2}$, \\ Mirco Grosser ${ }^{1,2}$, Rene Werner ${ }^{3}$, Tobias Knopp ${ }^{1,2}$ \\ ${ }^{1}$ Section for Biomedical Imaging, University Medical Center Hamburg-Eppendorf \\ ${ }^{2}$ Institute for Biomedical Imaging, Hamburg University of Technology \\ ${ }^{3}$ Department of Computational Neuroscience, University Medical Center \\ Hamburg-Eppendorf \\ ivo-matteo.baltruschat@tuhh.de
}

Die Magnetpartikelbildgebung (MPI) ist eine junge tomographische Bildgebungstechnik, die magnetische Nanopartikel mit einer hohen räumlichen und zeitlichen Auflösung quantitativ abbildet. Eine gängige Methode zur Rekonstruktion von MPI-Daten ist die Systemmatrix (SM)-basierte Rekonstruktion. Die komplexwertige SM wird in einer zeitaufwändigen Kalibrierungsmessung bestimmt. Die Anzahl der Voxel der SM beeinflusst direkt die Grösse des rekonstruierten Bildes, aber auch die Scanzeit - d.h. die Aufnahme einer $37^{3} \mathrm{SM}$ dauert etwa 32 Stunden im Vergleich zu einer $9^{3} \mathrm{SM}$, die etwa 37 Minuten dauert. In unserer Arbeit [1] haben wir untersucht, ob Deep Learning (DL) eingesetzt werden kann, um die Ergebnisse für die SM-Vervollständigung zu verbessern. Wir haben dafür ein Framework entwickelt, welches drei zentrale Schritte umfasst. Zunächst messen wir einen SM mit niedriger Auflösung auf einem spezifischen Abtastraster. Wir nutzen danach ein Faltungsnetzwerk, das wir 3d-System-Matrix-Wiederherstellungsnetzwerk (3d-SMRnet) nennen, um die hochauflösende SM wiederherzustellen. Wir haben das Modell so anpassen, dass es mit 3d-Eingangsdaten arbeitet, und wenden es auf jede Frequenzkomponente der SM an. Um unser Model zu trainieren, setzen wir eine neuartige Erweiterung der Verlustfunktion zur Behandlung komplexer Zahlen ein. Das 3d-SMRnet wird anhand eines öffentlichen MPI-Datensatzes evaluiert und im Vergleich zum derzeitigen Goldstandard - d.h. Compressed Sensing - erreicht es bessere Ergebnisse bei der SM-Vervollständigung und den rekonstruierten Bildern. Unsere neuartige Methode, die auf einem 3d-SMRnet und einem ComplexRGB-Verlust basiert, kann die Kalibrierungszeit im MPI erheblich verkürzen - d.h. 64-mal weniger Messpunkte im Vergleich zur ursprünglichen hochauflösenden Systemmatrix.

\section{Literatur}

1. Baltruschat IM, Szwargulski P, Griese F, et al. 3d-SMRnet: achieving a new quality of MPI system matrix recovery by deep learning. Proc MICCAI. 2020; p. 74-82. 